\title{
Subject Index Vol. 3, 1997
}

\section{Addiction Research}

Mental absorption 192 Meperidine 146 Methadone 123

- $\quad$ maintenance therapy 123

Methodology 199

Methylphenidate 49

Morphine 146

Naltrexone 110, 138 Natural conditions 150 Notifications 43

Opioid detoxification 146

- $\quad$ withdrawal 146

Overdose 87

Cocaine 49,93, 184 Comparability 199 Coping 37, 192 Cost-benefit analysis 160 Craving 110, 123

- $\quad$ measurement 116

Crime statistics 67

Criminal justice 67

Cross-national comparisons 75

Decision-making 67 Differential diagnosis 11 Drinking 75

habits 59 Drug(s) 192

abuse 99

Drug-related delinquency 67, 160 Dual diagnosis 164

Perceptions 87 Police discretion 67 Policing 67

Polydrug experimentation 184 Prescription of narcotics 160 Primary care 150 Principle of legality 67 Problem drinking 37

- $\quad$-, women 30

Professional treatment system for alcoholics 22 Psychopathology 123, 164, 173

Relapse 110

- $\quad$ prevention 129

Residential treatment 99

Reward system 110

Risk behaviours 87

Secondary prevention 150 Self-esteem 3 Self-help 22 Sensation seeking 192 Sense of coherence 99 Sexual abuse 3 Shame 3

Significant others 37 Social dynamics 59

- $\quad$ support 3

Substance abuse, intravenous 93

- $\quad$ use 83

Substance-induced psychosis 173 Substitution treatment 160

Translation validation 116 Treatment efficacy 99 
- $\quad$ seeking of female alcoholics 22

United Kingdom 43 University students 184

Women 3

\section{KAHGEK}

Fax + 41613061234 E-Mail karger@karger.ch wvirw.karger.com

(C) 1997 S. KargerAG, Basel 